\title{
Mutations in the TTN Gene are a Prognostic Factor for Patients with Lung Squamous Cell Carcinomas
}

\author{
Sheng Zou* \\ Jiayue $\mathrm{Ye} \mathbb{D}^{*}$ \\ Sheng $\mathrm{Hu}$ \\ Yiping Wei $(\mathbb{D}$ \\ Jianjun $\mathrm{Xu}$
}

Department of Cardiothoracic Surgery, the Second Affiliated Hospital of Nanchang University, Nanchang, Jiangxi Province, 330006, People's Republic of China

*These authors contributed equally to this work
Correspondence: Jianjun Xu; Yiping Wei Tel +8613907913526; +86/3687919322 Email xujianjun3526@163.com; weiyip2000@hotmail.com
Purpose: To analyze the relationship between titin (TTN) mutation gene and tumor mutational burden (TMB) and the with prognosis of lung squamous cell carcinomas (LUSC), and to explore the feasibility of $T T N$ as a potential prognostic marker of for LUSC.

Methods: We analyzed the somatic mutation landscape of LUSC samples using datasets obtained from The Cancer Genome Atlas (TCGA) and International Cancer Genome Consortium (ICGC) databases. Sequence data were divided into wild and mutant groups, and differences in TMB values between the groups compared using a Mann-Whitney $U$-test. The Kaplan Meier method was used to analyze the correlation between TTN mutation and LUSC prognosis, whereas CIBERSORT algorithm was used to calculate the degree of relative enrichment degree of among tumor-infiltrating lymphocytes in LUSC.

Results: Analysis of both datasets revealed high mutations in the TTN gene, with mutants exhibiting a significantly higher TMB value relative to the wild-type $(\mathrm{P}<0.001)$. Prognosis of the TTN mutant group in LUSC was significantly better than that of wild-type $(\mathrm{P}=0.009)$. Kaplan Meier curves showed that TTN mutation may be an independent prognostic factor in LUSC patients (HR: $0.64,95 \%$ CI $0.48-0.85, \mathrm{P}=0.001$ ), while GSEA analysis revealed that TTN mutation plays a potential role in the development of LUSC. Finally, analysis of LUSC immune microenvironment revealed that TTN mutation was significantly associated with enrichment of macrophages M1 ( $\mathrm{p}<0.05)$.

Conclusion: TTN mutation is associated with TMB, and is positively correlated with prognosis of LUSC. Therefore, this mutation may serve as a potential prognostic indicator of LUSC.

Keywords: TTN, lung squamous cell carcinomas, tumor mutation burden, prognostic marker, bioinformatics

\section{Introduction}

Lung cancer, the most common malignant tumor and one of the leading causes of cancer-related deaths worldwide, as evidenced by nearly 2 and 1.7 million new cases and deaths each year, respectively. ${ }^{1}$ Recent studies have shown that lung cancer is ranked second and first with regards to incidence and mortality, respectively, while its mortality rate is nearly twice as high as that of the prostate malignancy. ${ }^{2}$ Non-small cell lung cancer(NSCLC) accounts for $85 \%$ of all lung cancer cases, of which lung adenocarcinoma (LUAD) and lung squamous cell carcinomas (LUSC) are the most common tissue subtypes of lung cancer. ${ }^{3}$ LUSC, which is mainly caused by smoking, is the second largest type of non-small cell lung cancer. Previous studies have shown that although quitting smoking reduces 
the mortality rate of lung cancer to some extent, the incidence of LUSC is still high in many countries. ${ }^{4}$ In addition, despite the incidence of squamous cell carcinoma being lower than that of adenocarcinoma, the rate of gene mutations and treatment of the former is lower than that of the latter. Surgery is the main treatment therapy for patients with early LUSC, but efficacy of this approach is lower for those at late stages. In fact, the 5-year survival rate of platinum-based chemotherapy is less than $5 \%{ }^{5}$ In recent years, the survival rate of patients with advanced lung cancer has increased due to development of targeted drugs, although only a very small number of patients exhibit specific LUSC-driven mutations. ${ }^{6-8}$ Therefore, there is need to improve survival rate of patients with advanced LUSC.

The TTN gene, which is located on chromosome $2 \mathrm{q} 31$, comprises 364 exons. ${ }^{9,10}$ TTN is characterized by a huge and complex structure as well as a highly heterogeneous mutation process. ${ }^{11,12}$ Bioinformatics allows for simple analysis of the correlation between TTN with prognosis of all kinds of cancers, although the process has been associated with many false positive rates. At the same time, mutation of the TTN gene has been associated with myocardial or skeletal muscle disease, ${ }^{13-15}$ thereby casting uncertainty on the role of mutations in this gene in tumorigenesis. TTN's exon is the longest in the whole genome, and further comprises a huge number of mutation sites. ${ }^{16}$

TMB refers to the total number of non-synonymous mutation per million bases in the tumor tissue, and the average number of mutations in the tumor genome. Specifically, gene mutations are characterized by gene coding errors, base substitution, gene insertion and deletion errors. More somatic mutations imply production of more new antigens, which can stimulate more $\mathrm{T}$ cells to produce immune responses. ${ }^{17-19}$ Some studies have shown that higher TMB values indicates that the tumor can easily be detected by the immune system and become the target of tumor immunity, and the more effective it is for patients to receive immunotherapy. ${ }^{20-24}$

In this study, we downloaded and analyzed sequence data comprising somatic mutations in LUSC patients from The Cancer Genome Atlas (TCGA) and International Cancer Genome Consortium (ICGC) database. Our results revealed high rates of mutations in mutants from both datasets. Analysis of the relationship between these mutant genes and TMB with prognosis of LUSC revealed that genes that were closely related to prognosis were functionally enriched and combined with immune infiltration, suggesting their potential as new biomarkers. These may be potential targets for immunotherapy for patients with LUSC.

\section{Methods \\ Data Acquisition}

We obtained the corresponding data from the TCGA and ICGC databases, respectively. Somatic mutations and clinical data from an American cohort of lung squamous cell carcinoma patients were obtained from TCGA portal (http://por tal.gdc.cancer.gov/projects). Somatic mutations from an Korean cohort of lung squamous cell carcinoma patients were obtained from ICGC portal (http://dcc.icgc.org/ releases/current/Projects). Only patients with complete clinical data were included, while those with missing data, such as TNM staging, sex, age, and survival status, were excluded.

\section{Bioinformatics Analysis}

All statistical analyses were performed using packages implemented in $\mathrm{R}$ software version 4.0.3. Briefly, "VarScan" was used to detect and visualize LUSC mutant genes in MAF files. ${ }^{25}$ We extracted the mutated genes in each sample from the mutation databases of TCGA and ICGC, and genes with the top 30 mutation rates from both TCGA and ICGC databases were extracted by Perl, then subjected to "venn" reveal the intersection and obtain genes with high mutation frequency. According to the mutation of the gene, Mutant gene samples were divided into either a wild or mutant group, based on mutation profile, then the relationship between these crossover genes with TMB evaluated and visualized using the "ggpubr" package in R.

\section{Definition of TMB in LUSC}

The whole-exome sequencing (WES) data were used to calculate the TMB score in LUSC form TCGA and ICGC database. According to the statistics of all base substitutions and indels in the coding region of the target gene, silent mutations that failed to lead to amino acid changes were not counted in this study. The TMB value can be calculated from the total number of non-synonymous mutated bases, except the size of the exon. This method has been used to calculate TMB expression in LUSC samples obtained from TCGA database. ${ }^{26}$

\section{Gene Enrichment Analysis (GSEA) of TTN Mutation}

We performed gene enrichment analysis of TTN gene mutation and expression matrix data using GSEA software 
(v4.1.0). ${ }^{27}$ Gene expression set from the TCGA database represented the test, whereas data selected from "c2.cp. kegg.v7.2.symbols.gmt "in the MsigDB database represented the reference set. Each analysis was performed 1000 times, with data followed by $\mathrm{p}<0.01$ considered significantly enriched.

\section{Association Between Gene Mutations with Tumor-Infiltrating Immune Cells}

We evaluated the proportion of 22 immune cells in the tumor tissue using the CIBERSORT deconvolution algorithm@. Filtering condition was set to $\mathrm{p}<0.05$, and the immune cell proportion matrix data of each tumor sample is obtained. TTN was divided into two mutant and wild groups, then analyzed using the "limma" package to reveal differences in immune cells between the two groups. The relationship between gene mutations and tumor invasive immunity were presented as bar and violin graphs, generated using the "vioplot" package in R.

\section{Statistical Analysis}

All data were analyzed using packages implemented in $\mathrm{R}$ (v4.0.2). The relationship between gene mutations and TMB was analyzed using the Mann-Whitney $U$-test, using "Suvminer" for Survival Analysis. Profiles of gene mutations were assessed and used to divide the samples into mutant and wild groups, and the mutation data thereafter analyzed after being combined with clinical data. We used the Kaplan Meier method to generate survival curves, and applied log rank test for statistical significance $(p<0.05)$. Identification of statistically significant clinical characteristics among the patients was performed using univariate and multivariate Cox analyses. For all comparisons, a twotailed $p<0.05$ applied for statistical significance.

\section{Results}

\section{Characteristics of Somatic Mutations in LUSC}

A summary of the entire study and data analysis is shown in Figure 1. The downloaded mutation data from the TCGA database comprised 480 American LUSC samples, while that from the ICGC database belonged to 170 LUSC subjects from a Korean cohort. Each gene was counted and frequency of its mutations detected. The top 30 genes in the TCGA mutation database are shown in Figure 2A. Among them, TTN, TP53, CSMD3, MUC16 and RYR2 exhibited the highest mutation frequency. On the other hand, the top 30 genes in ICGC mutation database are shown in Figure 2B, of which TTN, TP53, MUC4, MUC16 and $\mathrm{ZFHX4}$ had the highest mutation frequency. Intersected mutant genes of the top 30 genes across both datasets revealed a total of 14 genes, Including TTN, TP53, $M U C 16$, and CSMD3, among others (Figure 3A).

\section{Relationship Between Gene Mutation with TMB}

We analyzed profiles of the 14 genes and divided the resulting dataset into wild and mutant groups. The TMB score in LUSC was between $0.02-58.2 / \mathrm{Mb}$, with a median value of $4.42 / \mathrm{Mb}$. Among the 14 frequently mutated genes, the mutant group exhibited a significantly higher TMB than the wild group $(p<0.001)$ (Figure 3B).

\section{Association Between Gene Mutation with Prognosis}

Kaplan Meier curves showed that TTN was positively correlated with prognosis of patients with LUSC. Among the 14 genes, mutations in TTN, and CSMD3 showed a significantly positively correlation with prognosis in LUSC patients $(\mathrm{p}<0.05)$ (Figure 4). Results from COX regression analysis revealed that only stage (HR: $1.65,95 \%$ CI,1.19-2.27, $\mathrm{p}=0.002$ ) and TTN mutations (HR: 0.64,95\% CI, $0.48 \sim 0.85, \mathrm{P}=0.001$ ) were statistically significant (Table 1 ).

\section{Gene Enrichment Pathway Analysis of TTN Mutations}

To explore the possible existence of single pathway for TTN mutation, we performed GSEA using the TCGA dataset, and found that glycosphingolipid biosynthesis ganglio series, intestinal immune network for iga production, leukocyte transendothelial migration, lysosome and vascular smooth muscle contraction was significantly enriched in TTN mutations (Figure 5). These are the possible pathways through which TTN mutations occur during development of LUSC.

\section{Association Between Tumor-Infiltrating Immune Cells with TTN Mutations in LUSC We applied the CIBERSORT algorithm to calculate immune infiltration of LUSC microenvironment and obtained 22 immune cells in each sample. Notably, $\mathrm{T}$ cells and macrophages accounted for the largest propor- tion of all LUSC samples (Figure 6A). Moreover, the TTN mutant group exhibited a higher proportion of Macrophages M1 than the wild group in LUSC}




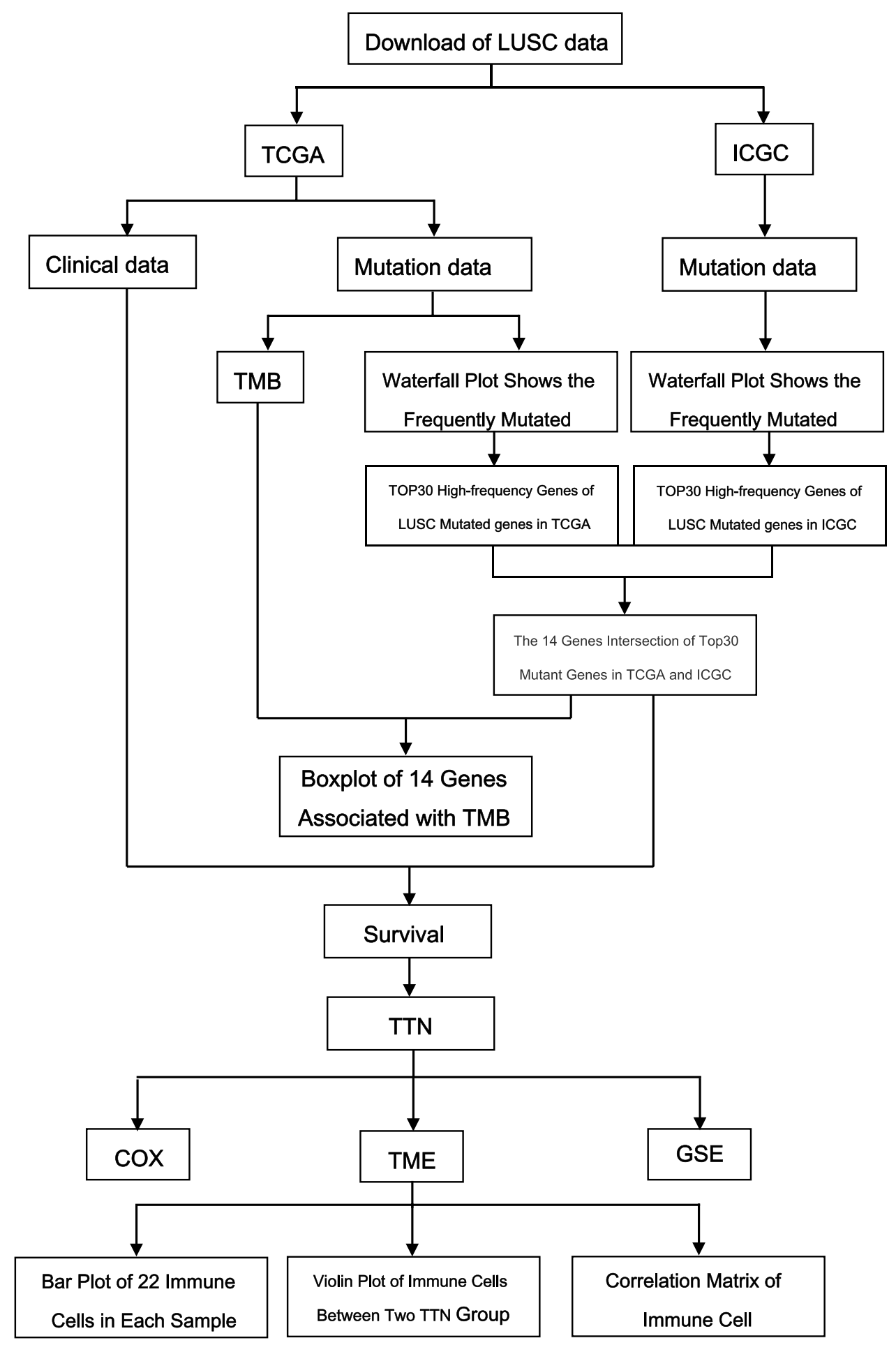

Figure I Analysis workflow of this study.

microenvironment $(\mathrm{P}<0.05)$ (Figure 6B). Furthermore, correlation results revealed a significant positive correlation between T cells CD8 and T cells CD4 memory activated, whereas mast cells resting and activated mast cells had the strongest negative correlation (Figure 6C).

\section{Discussion}

Although numerous studies have reported management of LUSC, mortality rates associated with the disease remain high. To date, clinical treatment and prognosis of LUSC are generally unsatisfactory, compared to 


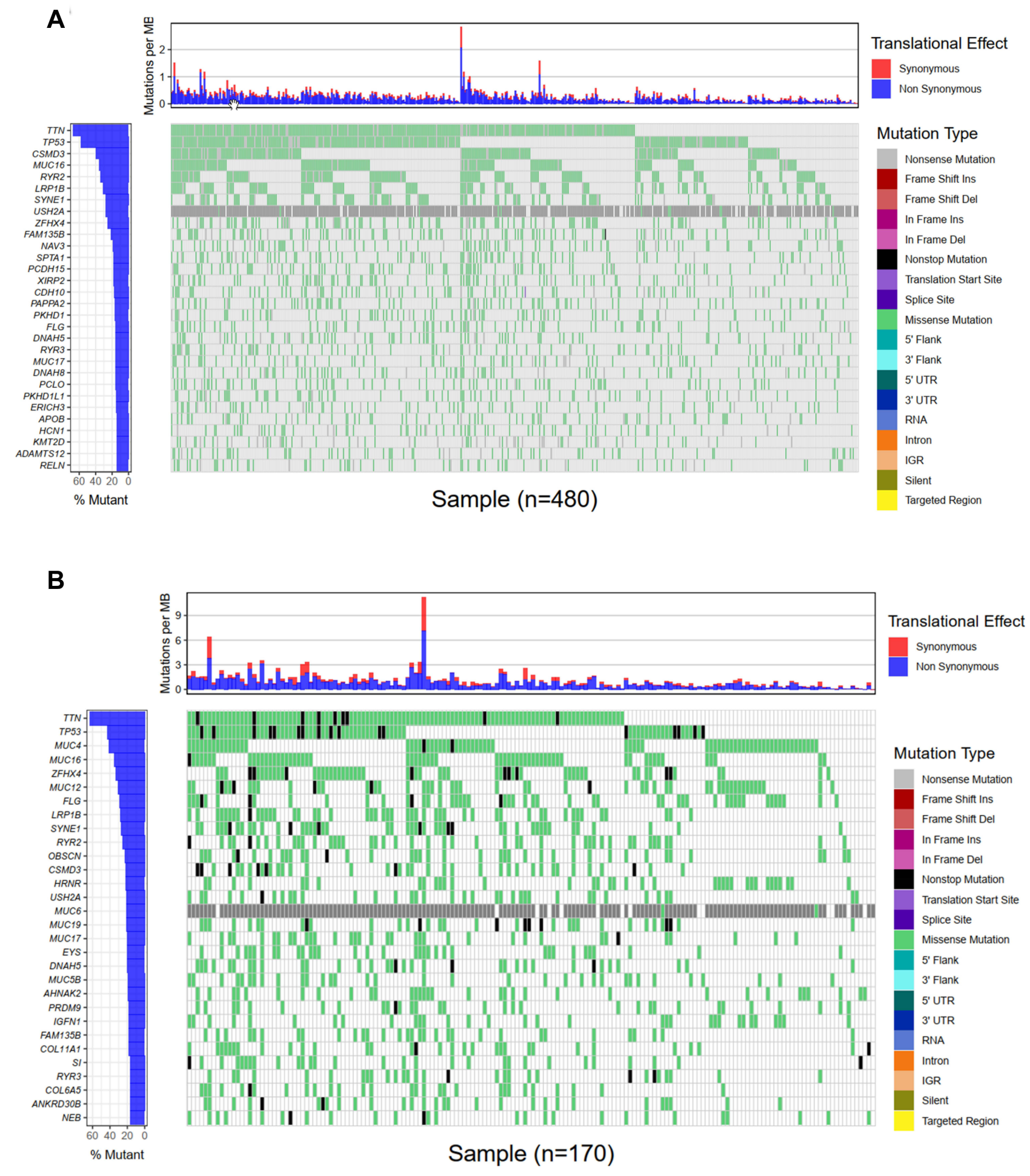

Figure 2 Landscapes of frequently mutated gene in LUSC. (A) Oncoplot depicts the frequently mutated genes in LUSC form TCGA cohort. The left panel shows mutation frequency, and genes are ordered by their mutation frequencies. The right panel presents different mutation types. (B) Waterfall plot displaying the frequently mutated genes in LUSC from the ICGC cohort. The left panel shows the genes ordered by their mutation frequencies. The right panel presents different mutation types.

early-diagnosed of LUAD, necessitating urgent development of therapeutic approaches to prevent loss of life. Advancement in high-throughput metrology technology all over the world has resulted in a large amount of data that can enable us to unravel the genetic changes caused by LUSC progression, thereby allowing identification of gene targets for early diagnosis, treatment and prognosis of the disease. In the present study, we analyzed somatic 


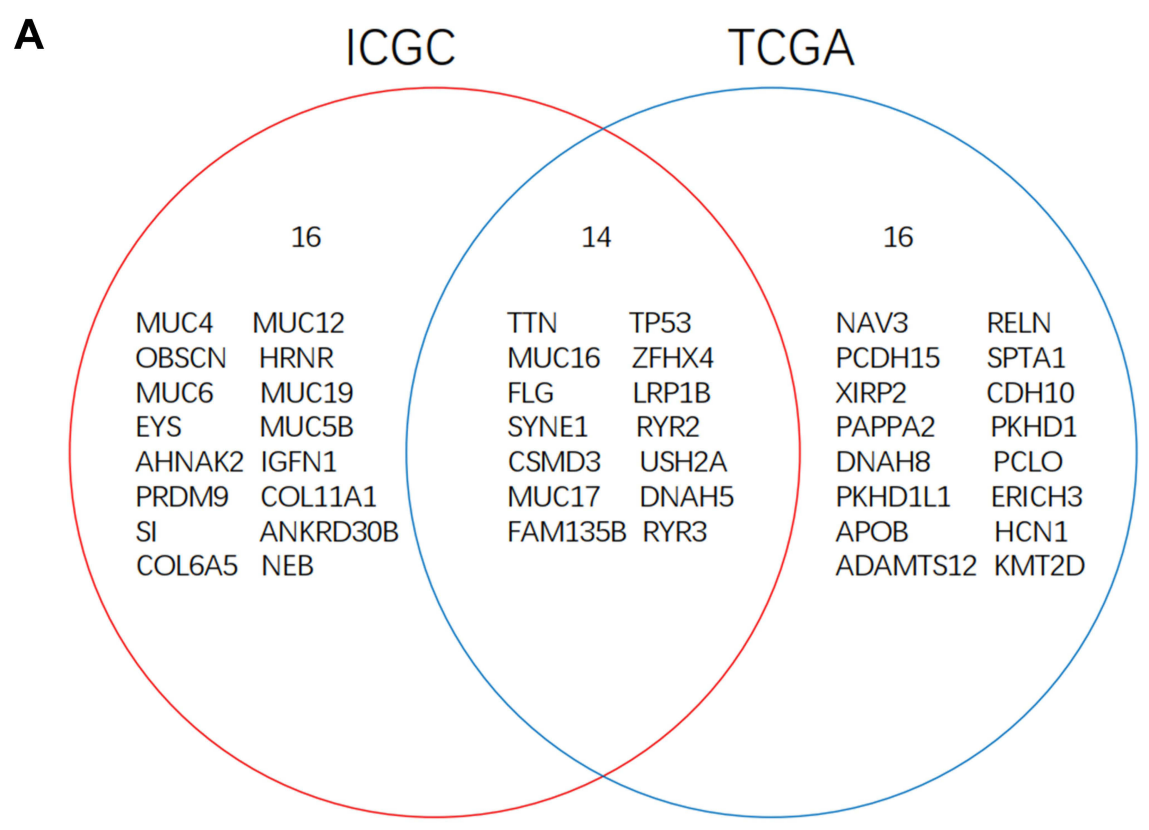

B Type 官 Wild 官 Mutation

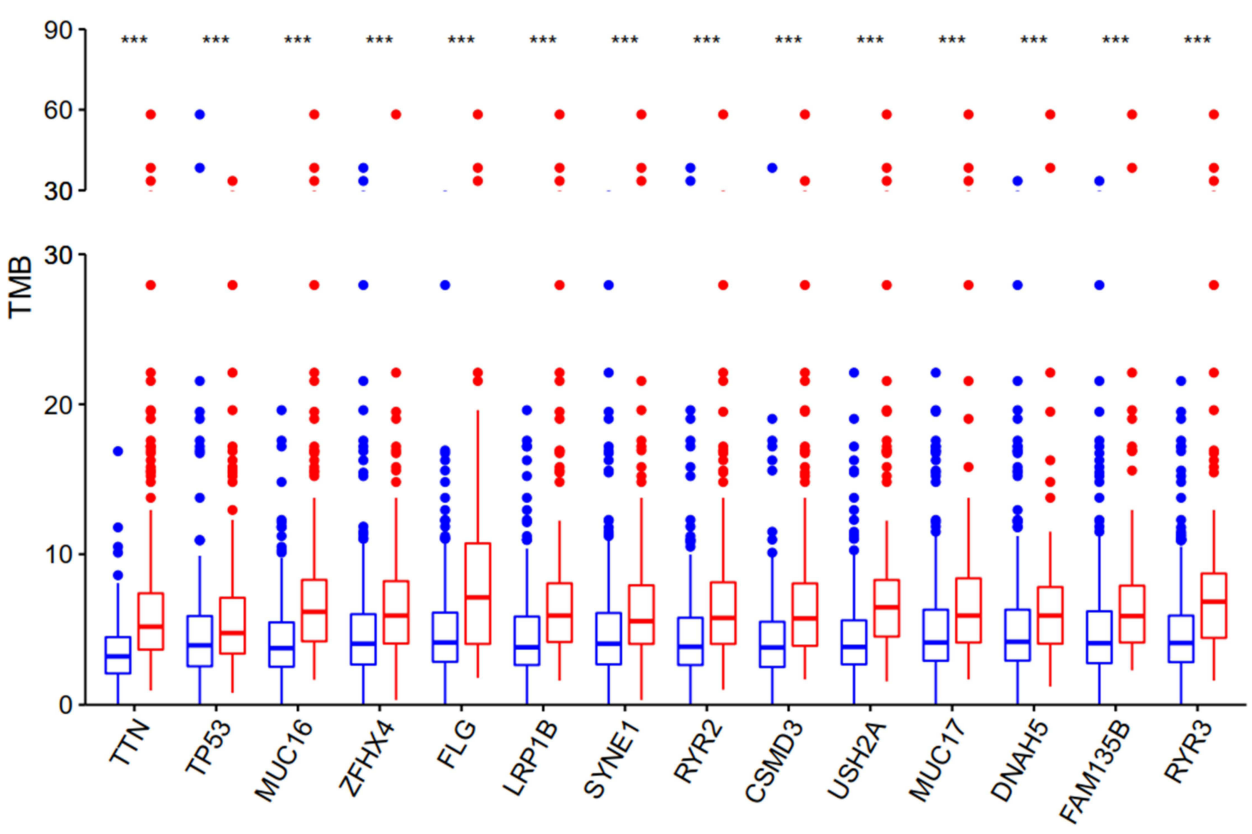

Figure 3 Gene mutations are associated with TMB. (A) Venn diagram shows 14 frequently mutated genes covered by the TCGA and ICGC cohorts. (B) Fourteen genes with high mutation frequency are associated with TMB $(* * * p<0.001)$.

Abbreviation: NS, no significance.

mutations in 480 and 170 Americans ad Korean LUSC patients, and found a high mutation frequency of TTN in datasets from both the TCGA and ICGC databases. In addition, our results indicated that TTN mutations had a positive prognostic value in LUSC patients, but was associated with TMB. Results from COX regression analysis showed that TTN mutation was statistically significant, and could be used as an independent risk factor in prognosis of patients with the disease. Finally, the proportion of macrophages M1 was significantly enriched in samples with TTN mutations in LUSC tumor microenvironment. 


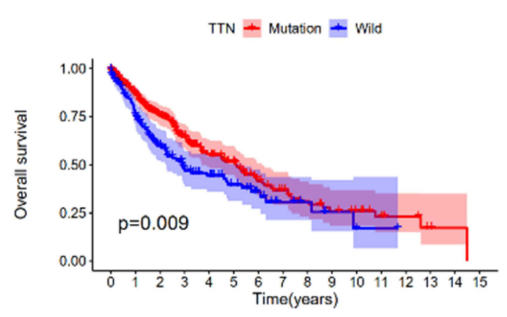

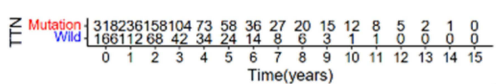

DNAH5 + Mutation - Wild

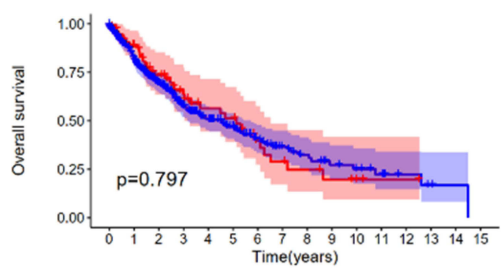

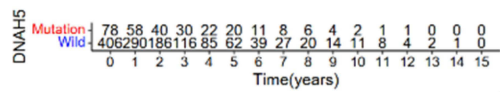

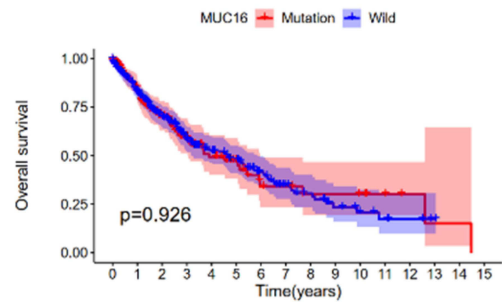

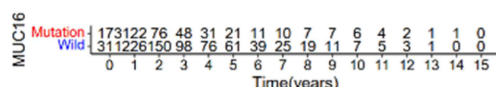

RYR3 - Mutation + Wild

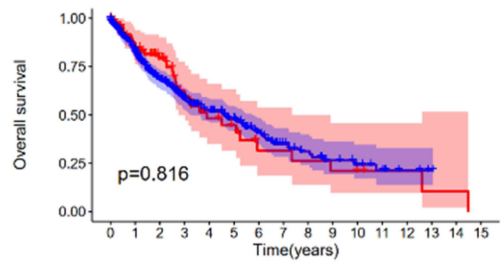

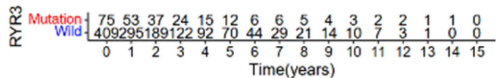

USH2A + Mutation + Wild

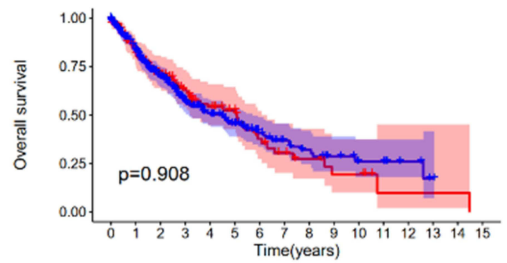

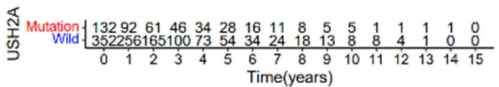

CSMD3 - Mutation + wild

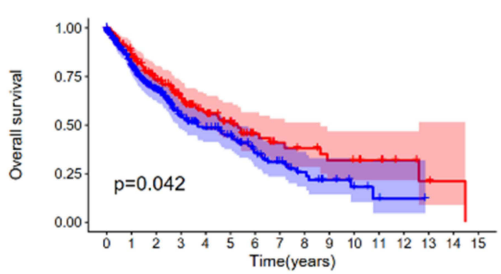

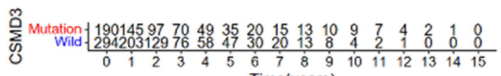

$\mathrm{FLG}=$ Mutation - wild

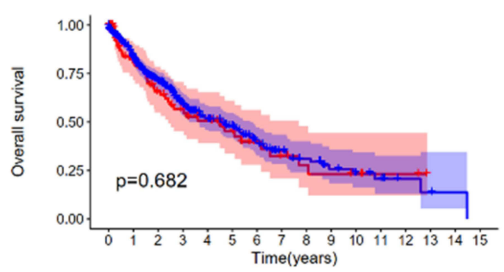

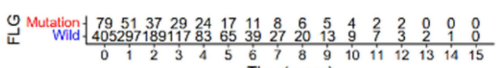

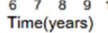

Muc17 + Mutation + wild

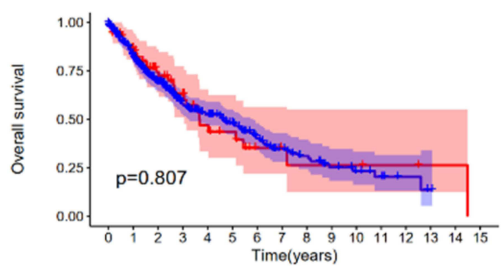

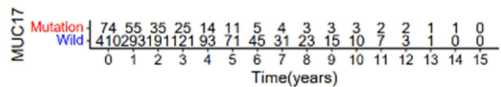

SYNE1 + Mutation + Wild

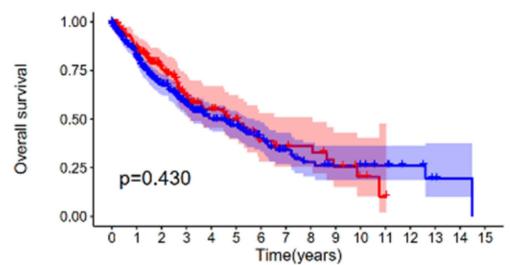

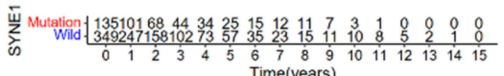

$\mathrm{ZFHX} 4-$ Mutation - Wild

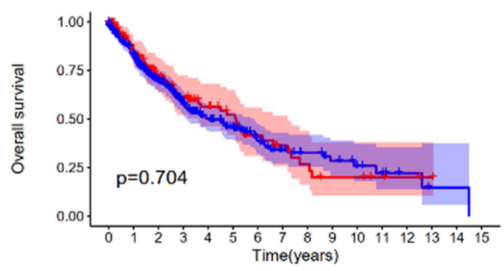

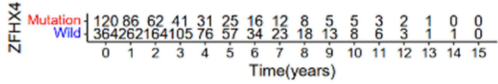

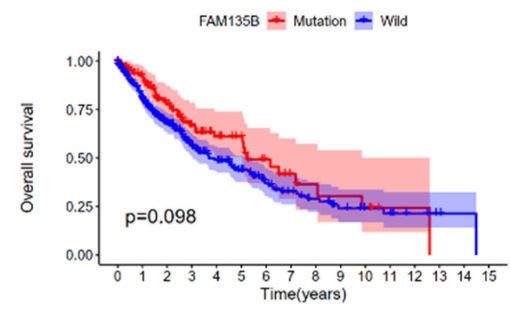

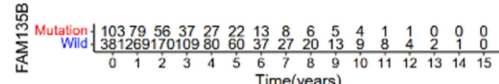

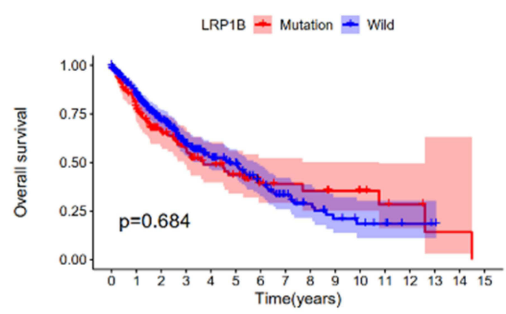

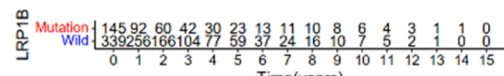

RYR2 + Mutation + Wild

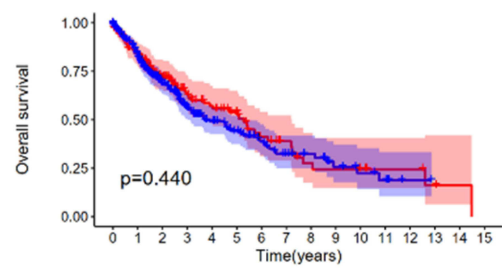

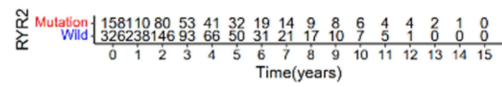

TP53 + Mutation + Wild

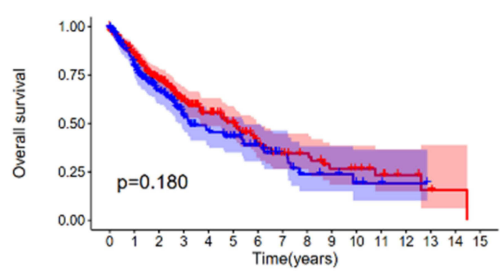

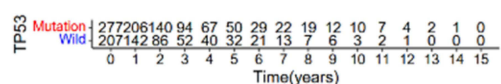

mutations and prognosis. The $\mathrm{p}$-value is shown each plot. 
Table I Univariate and Multivariate Overall Survival Analysis of LUSC Patients by the COX Proportional Hazards Model

\begin{tabular}{|l|l|l|l|l|}
\hline \multirow{2}{*}{ Factors } & \multicolumn{1}{|c|}{ Univariate } & \multicolumn{2}{|c|}{ Multivariate } \\
\cline { 2 - 5 } & HR (95\% Cl) & p-value & HR (95\% Cl) & P-value \\
\hline Age (year) $(<65, \geq 65)$ & $1.26(0.94 \sim 1.69)$ & 0.12 & \\
Gender (Male/Female) & $1.21(0.88 \sim 1.67)$ & 0.24 & \\
TNM classification (stagel and II, stage III and IV) & $1.58(1.15 \sim 2.18)$ & 0.005 & $1.65(1.19 \sim 2.27)$ & 0.002 \\
TMB (low, high) & $0.97(0.94 \sim 1.00)$ & 0.075 & \\
CSMD3 & $0.76(0.57 \sim 1.01)$ & 0.058 & \\
TTN & $0.66(0.50 \sim 0.87)$ & 0.003 & $0.64(0.48 \sim 0.85)$ & 0.001 \\
\hline
\end{tabular}

Titin is a structural protein in striated muscle. ${ }^{28}$ The mutation of this gene will mainly cause Cardiac Muscle Diseases and Skeletal Muscle Diseases. ${ }^{13-15,29}$ At present, the research of TTN mutation in tumor is very limited. Some studies have shown that the coding length of TTN is positively correlated with the frequency of gene mutation in solid tumors, and the predictive value of TTN gene mutation state is related to the length of its longest exon. ${ }^{16}$ The exon length of TTN is the longest in the whole genome, and the number of mutation sites in TTN exon is the most, which supports that the longer exon increases the possibility of accumulating more mutations. Not only TTN, but the second longest gene in the genome, MUC16, has been shown to be associated with higher TMB and good survival outcomes in patients with gastric cancer. ${ }^{30}$ The Muc16 gene is a gene that encodes the cancer antigen CA-125. In addition, some studies have shown that there is inflammatory tumor immune microenvironment (TIME) in patients with TTN mutation, which is characterized by abundant activated immune cells and high immune score. LUAD patients with TTN mutations showed high levels of immune antigens (immunogenicity), such as high TMB, high neoantigen load (nal) NAL and more non-synonymous mutations associated with DNA damage repair (DDR). ${ }^{31}$ These studies suggest that the better prognosis of LUAD patients with TTN mutations may be related to high immune antigens. Studies have shown that high levels of Tumor Necrosis Factor (TNF) can promote the secretion of a large number of IFN- $\gamma$ effector $\mathrm{T}$ cells. This cytokine further up-regulates the expression of MHC molecules by activating STAT1 signaling pathway. ${ }^{31}$ These studies show that TTN mutation can affect the prognosis of patients with LUAD, probably because the mutation leads to immune response, resulting in an increase in the number of targets that can produce immunotherapy or improve the response to some immune drugs, so as to achieve a higher survival effect. However, the effect of TTN mutation on the mechanism of tumor is not clear, and further verification is needed.

This is not the first time that gene mutation has been found to have a beneficial effect on the prognosis of tumors. Previous studies have suggested that patients with squamous cell carcinoma of the lung caused by mutations in the PIK3CA gene have a better prognosis than those without mutations. ${ }^{32}$ Another study also showed that in colon cancer, patients with harbored pole proof reading domain mutation had a better prognosis. This may be related to the mutation frequency of the gene. ${ }^{33}$ Considering that missense mutations occur in $85 \%$ of mutation cases, single nucleotide missense mutations usually lead to different amino acids encoded by codons. $^{34}$ Therefore, we infer that TTN nucleotide mutations may be caused by missense mutations, which promote a certain immune response or improve the response to certain drugs, thus achieving better survival. This study suggests that the mutation of TTN is beneficial to the prognosis of LUSC.

Tumor mutation load is a new biomarker, which plays an important role in tumor immunotherapy. At present, only a handful of studies have described TMB in lung squamous cell carcinoma. Results of the present study revealed a correlation between mutant LUSC gene with TMB, suggesting that it might be a driver for the disease, hence an ideal target for immunotherapies. Some studies have revealed a positive correlation between TMB and immunotherapy response, with high TMB levels implying better efficacy of immunotherapy. ${ }^{23,35}$ The Notably, more somatic mutations indicate increased antigen production, which activates more $\mathrm{T}$ cells to stimulate immune response, thereby enhancing efficacy. ${ }^{18}$ Some studies have shown that TMB can be used to evaluate efficacy 
A

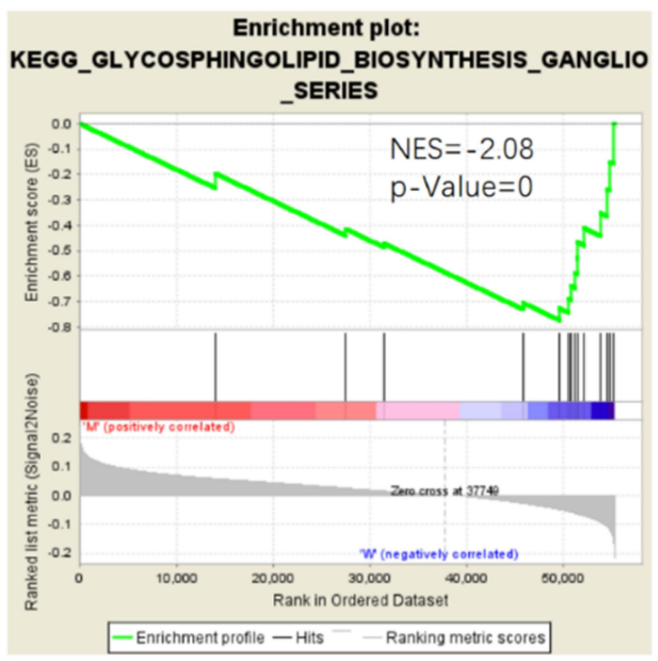

C
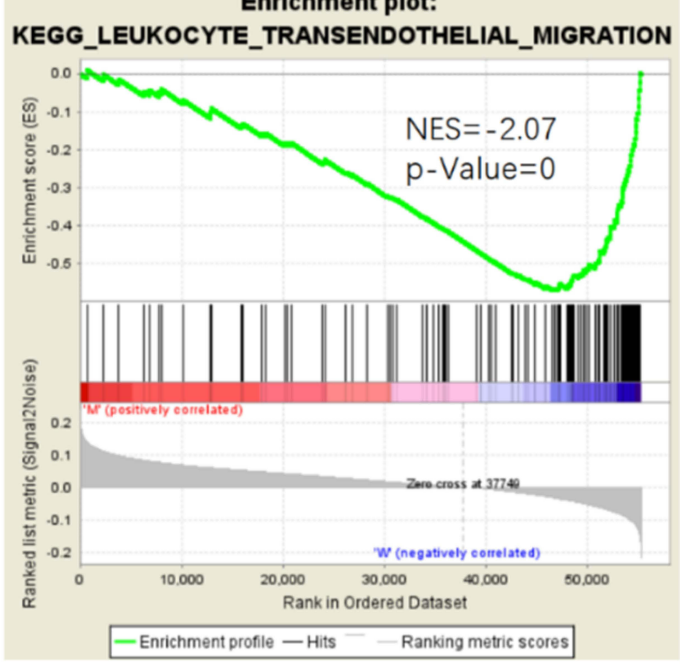

E

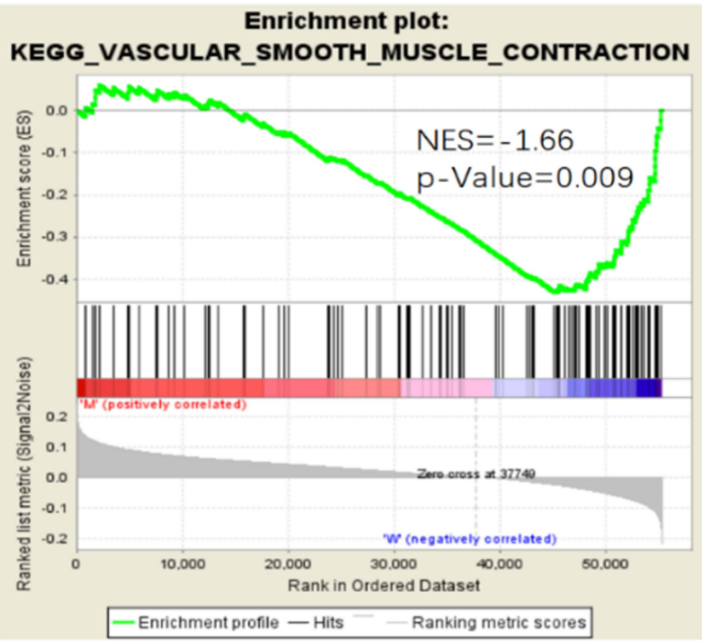

B

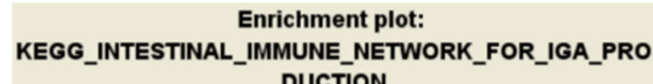
DUCTION

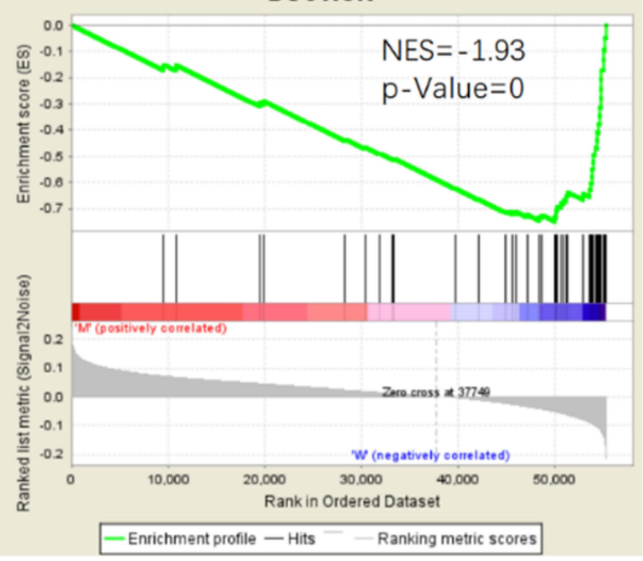

D

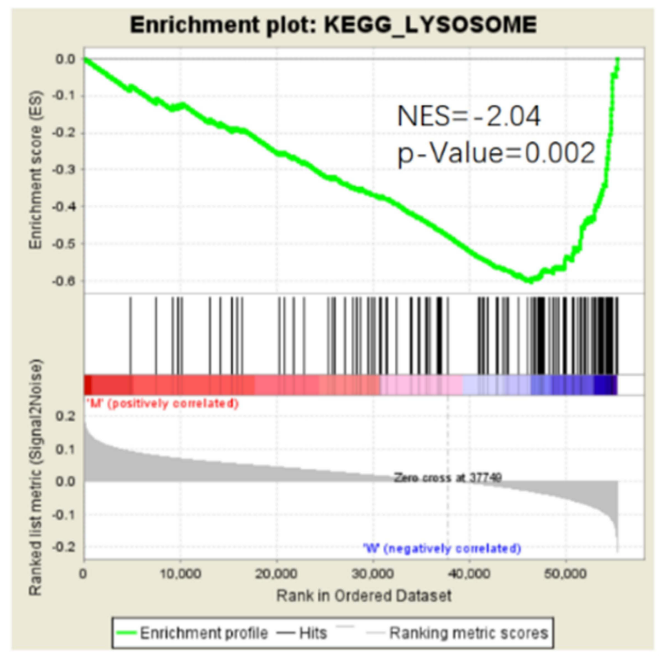

Figure 5 Significantly enriched pathways associated with TTN mutation. GSEA was performed with TCGA to explore the function role of TTN mutation. The GSEA analysis showed that samples with FAT3 mutation enriched in (A) glycosphingolipid biosynthesis ganglio series, (B) intestinal immune network for iga production, (C) leukocyte transendothelial migration, (D) lysosome, (E) vascular smooth muscle contraction. 
A

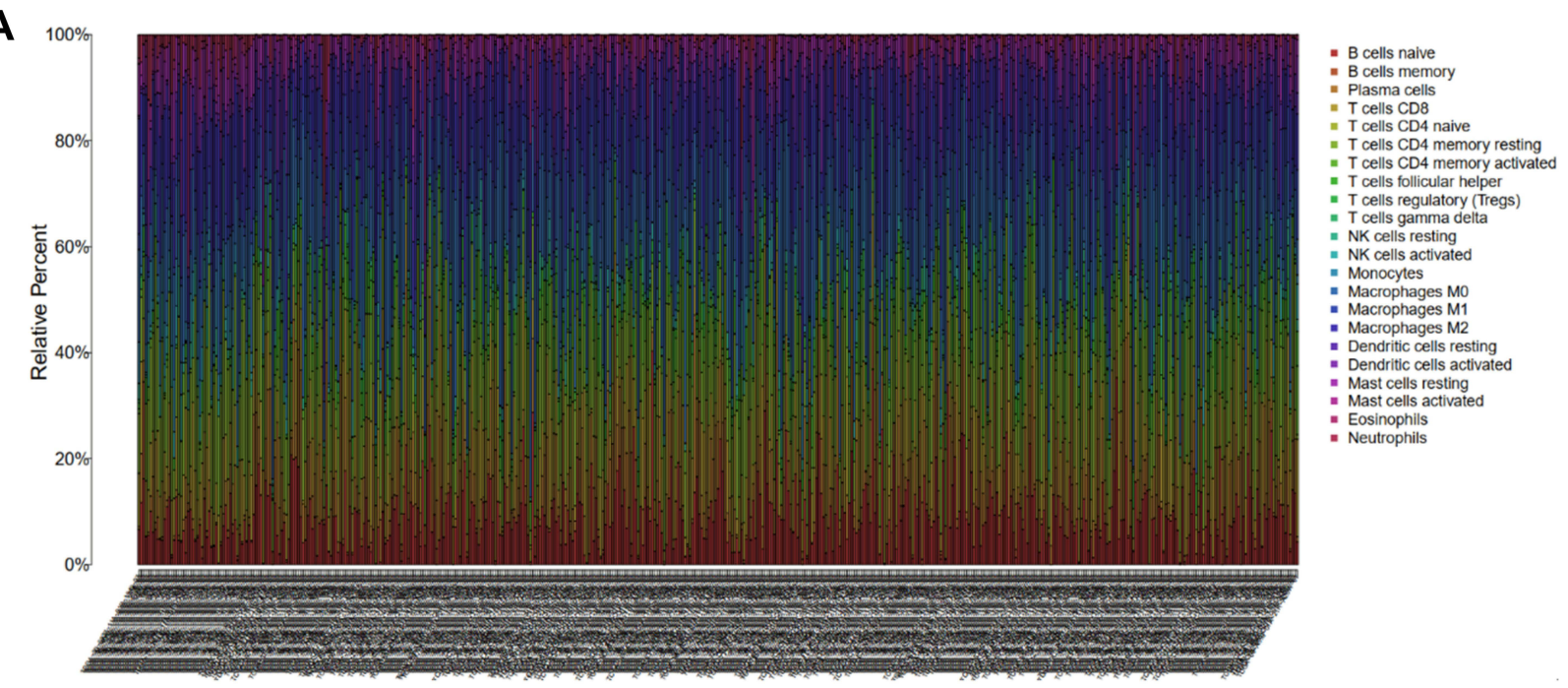

B

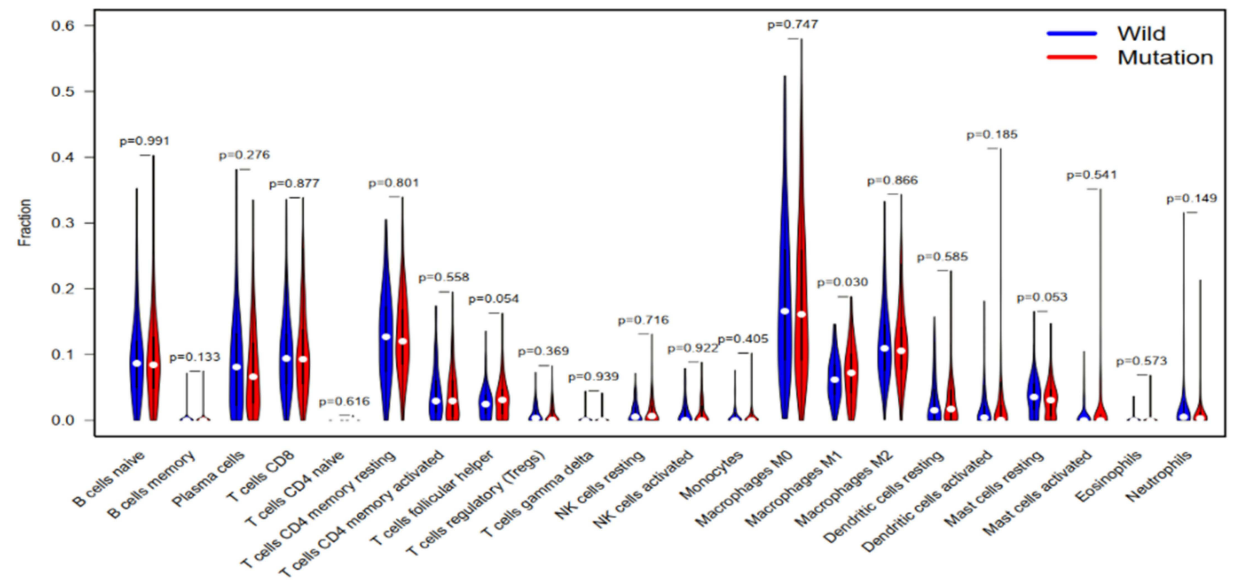

C

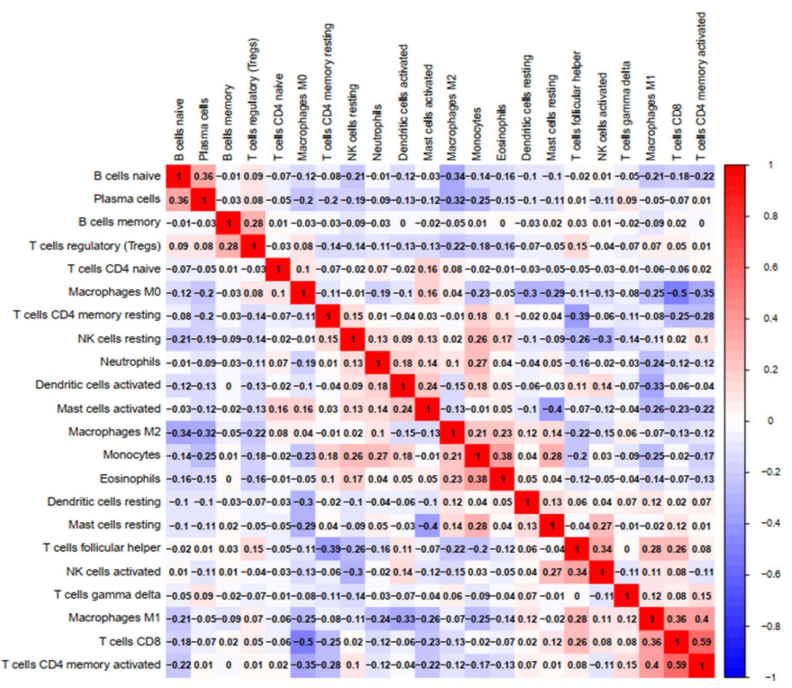

Figure 6 TTN mutation is correlated with tumor-infiltrating immune cells. (A) The stacked bar chart shows the distribution of 22 immune cells in each sample. (B) Violin plot displaying the differentially infiltrated immune cell between the TTN-mutant groups and the TTN-wild group, and blue represents the TTN-wild group, and red color represents TTN-mutation group. The p-value is shown in the figure. (C) Correlation matrix of immune cell proportions. The red color represents a positive correlation, and the blue color represents a negative correlation. 
of immunotherapy in both melanoma ${ }^{36}$ and NSCLC. ${ }^{37,38}$ Results from analysis of whole exon sequencing in the pan-cancer cohort of TCGA revealed that the TTN gene had the highest mutation rate and a high corresponding TMB value. Previous studies have demonstrated that most solid tumors exhibit high mutation probability of $T T N$, as well as elevated TMB which can subsequently be used to predict efficacy of immunotherapy in these tumors. ${ }^{16}$ Overall, these results suggest that TTN mutations in LUSC may drive the immune system against tumor cells, which is beneficial to immunotherapy.

GSEA analysis showed samples with TTN mutation enriched in "glycosphingolipid biosynthesis ganglio series"; "intestinal immune network for iga production"; "eukocyte transendothelial migration"; "lysosome" and "vascular smooth muscle contraction". In healthy adult nerve tissues, patients with high concentrations of the $\mathrm{b}$-series gangliosides and GD1b and GT1b have a better prognosis, which is related to the effect of gangliosides on cell proliferation and differentiation; $;{ }^{39-41}$ Iga is produced in the BALT of the respiratory mucosal system, ${ }^{42}$ and is therefore important in the fight against respiratory infection (including viral infections), which has been confirmed in bovine respiratory syncytial virus. ${ }^{43}$ IgA cells can have monocyte potential and antibacterial properties by rapidly secreting cytokines, which can be used as the regulation of inflammation; ${ }^{44}$ Leukocyte transendothelial migration is one of the most important steps in initiating inflammation and autoimmune response. ${ }^{45}$ Some studies have shown that Titin-truncating variant is related to decreased protein levels of the lysosomal protease. ${ }^{46}$ Another study has shown that vascular smooth muscle contraction and endocytosis were commonly involved in smoking and lung cancer. ${ }^{47}$ These studies can prove that these are the possible signal pathways in the tumorigenesis and development of LUSC patients with TTN mutation.

Macrophages have been shown to play a role in phagocytosis, cytotoxicity, secretion of pro-inflammatory factors, and have the ability to present antigens to $\mathrm{T}$ cells in the innate immune system. ${ }^{48}$ Notably, they have a unique ability to effect and penetrate tumors. ${ }^{49}$ Klichinsky et a ${ }^{50}$ found that macrophages and adenoviruses could promote the inflammatory tumor microenvironment and enhance the activity of tumor $\mathrm{T}$ cells in a mouse model after solid tumor transplantation. In fact, macrophages are enriched in the tumor microenvironment of most cancers, where they mainly promote invasion, formation of new blood vessels, metastasis and immunosuppression. ${ }^{51}$ Cancer-targeted antibody therapies have adopted application of macrophages as the key effectors, ${ }^{52}$ with activated macrophages found to play an important role in adaptive anti-tumor response. $^{48}$ Overall, these results affirm the important role played by macrophages in the immune microenvironment of tumors. Results of the present study revealed significantly enriched macrophage M1 in the TTN mutant, relative to the wildtype, group in LUSC microenvironment, suggesting a potential role in tumor immunity during LUSC progression.

This study still had some limitations. Firstly, we lacked clinical data on ICGC, hence we could not ascertain whether the lack of TTN was associated with to prognosis and the ripple effects on immune response. Secondly, our mutation data from ICGC only comprised Korean LUSC patients, which may be ethnically different from those of American LUSC patients in TCGA. Finally, we only applied bioinformatics to study TTN, owing to the complexity of tumor immunotherapy. In this paper, there is a lack of analysis of TTN exon mutations, so we cannot rule out exon non-functional region mutations, and further functional experiments are needed to verify TTN.

\section{Conclusion}

Analysis of somatic mutation data using datasets from TCGA and ICGC databases revealed high frequency mutations in the TTN gene in LUSC patients. These mutations were correlated with TMB. These mutations were positively correlated with LUSC. Results from COX regression analysis revealed that TTN mutation was an independent risk factor for LUSC development, suggesting that it can be used as a prognostic indicator for this disease, hence a target for future immunotherapies.

\section{Abbreviations}

TTN, titin; TMB, tumor mutation burden; LUSC, lung squamous cell carcinomas; LUAD, lung adenocarcinoma; NSCLC, non-small cell lung cancer; TCGA, The Cancer Genome Atlas; ICGC, International Cancer Genome Consortium; GESA, Gene Set Enrichment Analysis.

\section{Ethics Statement}

Our study did not involve any experiment on humans or animals; therefore, no ethical approval was obtained.

\section{Disclosure}

The authors report no conflicts of interest in this work. 


\section{References}

1. Bray F, Ferlay J, Soerjomataram I, Siegel RL, Torre LA, Jemal A. Global cancer statistics 2018: GLOBOCAN estimates of incidence and mortality worldwide for 36 cancers in 185 countries. CA Cancer J Clin. 2018;68(6):394-424. doi:10.3322/caac.21492

2. Siegel RL, Miller KD, Fuchs HE, Jemal A. Cancer Statistics, 2021. CA Cancer J Clin. 2021;71(1):7-33. doi:10.3322/caac.21654

3. Molina JR, Yang P, Cassivi SD, Schild SE, Adjei AA. Non-small cell lung cancer: epidemiology, risk factors, treatment, and survivorship. Mayo Clin Proc. 2008;83(5):584-594. doi:10.1016/S0025-6196(11)60735-0

4. Islami F, Torre LA, Jemal A. Global trends of lung cancer mortality and smoking prevalence. Transl Lung Cancer Res. 2015;4 (4):327-338. doi:10.3978/j.issn.2218-6751.2015.08.04

5. Rizvi NA, Hellmann MD, Brahmer JR, et al. Nivolumab in combination with platinum-based doublet chemotherapy for first-line treatment of advanced non-small-cell lung cancer. J Clin Oncol. 2016;34 (25):2969-2979. doi:10.1200/JCO.2016.66.9861

6. Reck M, Rabe KF. Precision diagnosis and treatment for advanced non-small-cell lung cancer. $N$ Engl J Med. 2017;377(9):849-861. doi:10.1056/NEJMra1703413

7. Hu Z, Bi G, Sui Q, et al. Analyses of multi-omics differences between patients with high and low PD1/PDL1 expression in lung squamous cell carcinoma. Int Immunopharmacol. 2020;88:106910. doi:10.1016/j.intimp.2020.106910

8. Tanegashima T, Togashi Y, Azuma K, et al. Immune Suppression by PD-L2 against Spontaneous and Treatment-Related Antitumor Immunity. Clin Cancer Res. 2019;25(15):4808-4819. doi:10.1158/ 1078-0432.CCR-18-3991

9. Hackman P, Vihola A, Haravuori H, et al. Tibial muscular dystrophy is a titinopathy caused by mutations in TTN, the gene encoding the giant skeletal-muscle protein titin. Am J Hum Genet. 2002;71 (3):492-500. doi:10.1086/342380

10. Bang ML, Centner T, Fornoff F, et al. The complete gene sequence of titin, expression of an unusual approximately $700-\mathrm{kDa}$ titin isoform, and its interaction with obscurin identify a novel Z-line to I-band linking system. Circ Res. 2001;89(11):1065-1072. doi:10.1161/hh2301.100981

11. Kim Y-A, Madan S, Przytycka TM. WeSME: uncovering mutual exclusivity of cancer drivers and beyond. Bioinformatics. 2017;33 (6):814-821. doi:10.1093/bioinformatics/btw242

12. Hofree M, Shen JP, Carter H, Gross A, Ideker T. Network-based stratification of tumor mutations. Nat Methods. 2013;10 (11):1108-1115. doi:10.1038/nmeth.2651

13. Schafer S, de Marvao A, Adami E, et al. Titin-truncating variants affect heart function in disease cohorts and the general population. Nat Genet. 2017;49(1):46-53. doi:10.1038/ng.3719

14. Watanabe $\mathrm{H}$, Atsuta N, Hirakawa A, et al. A rapid functional decline type of amyotrophic lateral sclerosis is linked to low expression of TTN. J Neurol Neurosurg Psychiatry. 2016;87(8):851-858. doi:10.1136/jnnp-2015-311541

15. Herman DS, Lam L, Taylor MRG, et al. Truncations of titin causing dilated cardiomyopathy. N Engl J Med. 2012;366(7):619-628. doi:10.1056/NEJMoa1110186

16. Jia Q, Wang J, He N, He J, Zhu B. Titin mutation associated with responsiveness to checkpoint blockades in solid tumors. JCI Insight. 2019;4(10):10. doi:10.1172/jci.insight.127901

17. Yarchoan M, Hopkins A, Jaffee EM. Tumor Mutational Burden and Response Rate to PD-1 Inhibition. $N$ Engl J Med. 2017;377 (25):2500-2501. doi:10.1056/NEJMc1713444

18. Addeo A, Friedlaender A, Banna GL, Weiss GJ. TMB or not TMB as a biomarker: that is the question. Crit Rev Oncol Hematol. 2021;163:103374. doi:10.1016/j.critrevonc.2021.103374

19. Jardim DL, Goodman A, de Melo Gagliato D, Kurzrock R. The Challenges of Tumor Mutational Burden as an Immunotherapy Biomarker. Cancer Cell. 2021;39(2):154-173. doi:10.1016/j. ccell.2020.10.001
20. Büttner R, Longshore JW, López-Ríos F, et al. Implementing TMB measurement in clinical practice: considerations on assay requirements. ESMO Open. 2019;4(1):e000442. doi:10.1136/esmoopen-2018-000442

21. Liu L, Bai X, Wang J, et al. Combination of TMB and CNA stratifies prognostic and predictive responses to immunotherapy across metastatic cancer. Clin Cancer Res. 2019;25(24):7413-7423. doi:10.1158/ 1078-0432.CCR-19-0558

22. Samstein RM, Lee C-H, Shoushtari AN, et al. Tumor mutational load predicts survival after immunotherapy across multiple cancer types. Nat Genet. 2019;51(2):202-206. doi:10.1038/s41588-018-0312-8

23. Chan TA, Yarchoan M, Jaffee E, et al. Development of tumor mutation burden as an immunotherapy biomarker: utility for the oncology clinic. Ann Oncol. 2019;30(1):44-56. doi:10.1093/annonc/mdy495

24. Hellmann MD, Nathanson T, Rizvi H, et al. Genomic features of response to combination immunotherapy in patients with advanced non-small-cell lung cancer. Cancer Cell. 2018;33:5. doi:10.1016/j. ccell.2018.03.018

25. Mayakonda A, Lin D-C, Assenov Y, Plass C, Koeffler HP. Maftools: efficient and comprehensive analysis of somatic variants in cancer. Genome Res. 2018;28(11):1747-1756. doi:10.1101/gr.239244.118

26. Chalmers ZR, Connelly CF, Fabrizio D, et al. Analysis of 100,000 human cancer genomes reveals the landscape of tumor mutational burden. Genome Med. 2017;9(1):34. doi:10.1186/s13073-017-0424-2

27. Subramanian A, Tamayo P, Mootha VK, et al. Gene set enrichment analysis: a knowledge-based approach for interpreting genome-wide expression profiles. Proc Natl Acad Sci U S A. 2005;102 (43):15545-15550. doi:10.1073/pnas.0506580102

28. Ciferri A, Crumbliss AL. The assembling and contraction mechanisms of striated muscles. Front Chem. 2018;6:570. doi:10.3389/ fchem.2018.00570

29. Ceyhan-Birsoy O, Agrawal PB, Hidalgo C, et al. Recessive truncating titin gene, TTN, mutations presenting as centronuclear myopathy. Neurology. 2013;81(14):1205-1214. doi:10.1212/WNL.0b013e3182a6ca62

30. Li X, Pasche B, Zhang W, Chen K. Association of MUC16 mutation with tumor mutation load and outcomes in patients with gastric cancer. JAMA Oncol. 2018;4(12):1691-1698. doi:10.1001/jamaoncol.2018.2805

31. Wang Z, Wang C, Lin S, Yu X. Effect of TTN mutations on immune microenvironment and efficacy of immunotherapy in lung adenocarcinoma patients. Front Oncol. 2021;11:725292. doi:10.3389/ fonc. 2021.725292

32. McGowan M, Hoven AS, Lund-Iversen M, et al. PIK3CA mutations as prognostic factor in squamous cell lung carcinoma. Lung Cancer. 2017;103:52-57. doi:10.1016/j.lungcan.2016.11.018

33. Domingo E, Freeman-Mills L, Rayner E, et al. Somatic POLE proofreading domain mutation, immune response, and prognosis in colorectal cancer: a retrospective, pooled biomarker study. Lancet Gastroenterol Hepatol. 2016;1(3):207-216. doi:10.1016/S2468-1253(16)30014-0

34. Minde DP, Anvarian Z, Rüdiger SG, Maurice MM. Messing up disorder: how do missense mutations in the tumor suppressor protein APC lead to cancer? Mol Cancer. 2011;10(1):101. doi:10.1186/1476-4598-10-101

35. Snyder A, Makarov V, Merghoub T, et al. Genetic basis for clinical response to CTLA-4 blockade in melanoma. $N$ Engl J Med. 2014;371 (23):2189-2199. doi:10.1056/NEJMoa1406498

36. Rooney MS, Shukla SA, Wu CJ, Getz G, Hacohen N. Molecular and genetic properties of tumors associated with local immune cytolytic activity. Cell. 2015;160(1-2):48-61. doi:10.1016/j.cell.2014.12.033

37. Chen Y, Liu Q, Chen Z, et al. PD-L1 expression and tumor mutational burden status for prediction of response to chemotherapy and targeted therapy in non-small cell lung cancer. J Exp Clin Cancer Res. 2019;38(1):193. doi:10.1186/s13046-019-1192-1

38. Chen H, Chong W, Wu Q, Yao Y, Mao M, Wang X. Association of mutation with tumor mutation burden and outcomes in melanoma and non-small cell lung cancer patients treated with immune check-point blockades. Front Immunol. 2019;10:1113. doi:10.3389/fimmu.20 19.01113 
39. Schengrund CL, Repman MA, Shochat SJ. Ganglioside composition of human neuroblastomas. Correlation with prognosis. A Pediatric Oncology Group Study. Cancer. 1985;56(11):2640-2646. doi:10.1002/1097-0142(19851201)56:11<2640::AID-

CNCR2820561118>3.0.CO;2-W

40. Hettmer S, Malott C, Woods W, Ladisch S, Kaucic K. Biological stratification of human neuroblastoma by complex "B" pathway ganglioside expression. Cancer Res. 2003;63(21):7270-7276.

41. Olsson M, Beck S, Kogner P, Martinsson T, Carén H. Genome-wide methylation profiling identifies novel methylated genes in neuroblastoma tumors. Epigenetics. 2016;11(1):74-84. doi:10.1080/ 15592294.2016.1138195

42. Chase C, Kaushik RS. Mucosal immune system of cattle: all immune responses begin here. Vet Clin North Am Food Anim Pract. 2019;35 (3):431-451. doi:10.1016/j.cvfa.2019.08.006

43. Kolb EA, Buterbaugh RE, Rinehart CL, et al. Protection against bovine respiratory syncytial virus in calves vaccinated with adjuvanted modified live vaccine administered in the face of maternal antibody. Vaccine. 2020;38(2):298-308. doi:10.1016/j.vaccine. 20 19.10.015

44. Gommerman JL, Rojas OL, Fritz JH. Re-thinking the functions of IgA(+) plasma cells. Gut Microbes. 2014;5(5):652-662. doi:10.4161/ 19490976.2014.969977

45. Getter T, Margalit R, Kahremany S, et al. Novel inhibitors of leukocyte transendothelial migration. Bioorg Chem. 2019;92:103250. doi:10.1016/j.bioorg.2019.103250
46. Zhou J, Ng B, Ko NSJ, et al. Titin truncations lead to impaired cardiomyocyte autophagy and mitochondrial function in vivo. Hum Mol Genet. 2019;28(12):1971-1981. doi:10.1093/hmg/ddz033

47. Fang X, Netzer M, Baumgartner C, Bai C, Wang X. Genetic network and gene set enrichment analysis to identify biomarkers related to cigarette smoking and lung cancer. Cancer Treat Rev. 2013;39 (1):77-88. doi:10.1016/j.ctrv.2012.06.001

48. Tang-Huau T-L, Gueguen P, Goudot C, et al. Human in vivo-generated monocyte-derived dendritic cells and macrophages cross-present antigens through a vacuolar pathway. Nat Commun. 2018;9(1):2570. doi:10.1038/s41467-018-04985-0

49. Ritchie D, Mileshkin L, Wall D, et al. In vivo tracking of macrophage activated killer cells to sites of metastatic ovarian carcinoma. Cancer Immunol Immunother. 2007;56(2):155-163. doi:10.1007/s00262-0060181-3

50. Klichinsky M, Ruella M, Shestova O, et al. Human chimeric antigen receptor macrophages for cancer immunotherapy. Nat Biotechnol. 2020;38(8):947-953. doi:10.1038/s41587-020-0462-y

51. Noy R, Pollard JW. Tumor-associated macrophages: from mechanisms to therapy. Immunity. 2014;41(1):49-61. doi:10.1016/j. immuni.2014.06.010

52. Weiskopf K, Weissman IL. Macrophages are critical effectors of antibody therapies for cancer. MAbs. 2015;7(2):303-310. doi:10.1080/19420862.2015.1011450
International Journal of General Medicine

\section{Publish your work in this journal}

The International Journal of General Medicine is an international, peer-reviewed open-access journal that focuses on general and internal medicine, pathogenesis, epidemiology, diagnosis, monitoring and treatment protocols. The journal is characterized by the rapid reporting of reviews, original research and clinical studies

\section{Dovepress}

across all disease areas. The manuscript management system is completely online and includes a very quick and fair peer-review system, which is all easy to use. Visit http://www.dovepress.com/ testimonials.php to read real quotes from published authors. 